\title{
Comparison of Various Abdominal Obesity Measures for Predicting Metabolic Syndrome, Diabetes, Nephropathy, and Dyslipidemia
}

\author{
Sikandar Hayat Khan1, Rahat Shahid2, Nadeem Fazal3 and Aamir ljaz ${ }^{4}$ \\ 1 Department of Pathology / 2 Radiology / 3 Medicine, PNS Hafeez, Islamabad, Pakistan \\ ${ }^{4}$ Department of Chemical Pathology, Armed Forces Institute of Pathology (AFIP), Rawalpindi, Pakistan
}

\begin{abstract}
Objective: To evaluate abdominal volume index (AVI), body roundness index (BRI), body adiposity index (BAI), a body shape index (ABSI) and conicity index (C-Index) for differences in subjects with or without metabolic syndrome, diabetes, nephropathy, and dyslipidemia; and secondly, to evaluate the diagnostic performance through measuring area under curve (AUC) by ROC curve analysis for new and conventional obesity measures in diagnosing metabolic syndrome.

Study Design: Cross-sectional analytical study.

Place and Duration of Study: PNS Hafeez Hospital, Islamabad, from January 2016 to December 2018.

Methodology: Baseline anthropometric measures including BMI, WHpR, WHtR, AVI, BRI, BAI, ABSI and C-Index were measured for 232 subjects along with measurement of various biochemical parameters. Differences among subjects with and without metabolic syndrome, diabetes, nephropathy, and groups based upon insulin resistance were noted. ROC curve analysis was utilised to measure AUC for all anthropometric measures for diagnosing metabolic syndrome.

Results: Pearson's correlation between obesity measures and lipid indices suggested highest correlation for AVI for most lipid indices followed by WHpR and WHtR. Mean AUC for obesity measures were greater than 0.80 for WHtR and AVI, followed by other parameters. The least AUC i.e. 0.320, was observed for ABSI. The differences between various anthropometric measures for groups based upon metabolic syndrome, diabetes, nephropathy, and insulin resistance remain variable indicating that each anthropometric index may depict a different aspect of the metabolic risk.

Conclusion: WHtR and AVI showed the highest AUC to diagnose metabolic syndrome and were better associated with metabolic diseases.
\end{abstract}

Key Words: Abdominal volume index (AVI), Body roundness index (BRI), Body adiposity index (BAI), A body shape index (ABSI), Conicity index (C-Index), Waist to hip ratio (WHpR), Waist to height ratio (WHtR).

How to cite this article: Khan SH, Shahid R, Fazal N, ljaz A. Comparison of various abdominal obesity measures for predicting metabolic syndrome, diabetes, nephropathy, dyslipidemia. J Coll Physicians Surg Pak 2019; 29(12):1159-1164.

\section{INTRODUCTION}

Though the factors involved may range from genetics to dietary patterns and inactive lifestyle, it still remains crucial to interpret the nature and pattern of obesity. 1 Visceral adiposity is related to higher metabolic morbidity and mortality due to associated higher inflammation, unlike the adiposity appearing in other areas of the body. ${ }^{2}$

However, related controversies need to be appreciated. Firstly, body mass index (BMI) relies on weight which is mainly contributed by mass rather than fat deposits as seen among people with muscular bodies. ${ }^{3}$ Waist and Waist-to-hip ratio (WHpR) specifically address abdominal pattern of obesity; however, these measurements are

Correspondence to: Dr. Sikandar Hayat Khan, Department of

Pathology, PNS Hafeez, E-8 Sector, Islamabad, Pakistan

E-mail:sik_cpsp@yahoo.com

Received: April 04, 2019; Revised: September 07, 2019;

Accepted: October 07, 2019 confounded by body frame, gender, race, variability of denominator and issues related with non-uniformity of measurements. 4 Waist-to-height ratio (WHtR) has shown a constant denominator in terms of fixed height in adults and has performed better than above parameters in the studies. ${ }^{5}$ Recent data highlights incorporation of newer obesity measures and research to have better obesity measures portraying risk associated visceral fat. ${ }^{6}$

New obesity measures targeting specifically visceral fat have, therefore, surfaced. Abdominal volume index (AVI) is a measure which has performed superior to waist circumference and other conventional anthropometric indices predicting diabetes. ${ }^{7}$ Body roundness index (BRI) and BMI are also demonstrated to be superior to other obesity measures in depicting cardiometabolic abnormalities. ${ }^{8} \mathrm{~A}$ body shape index (ABSI) was also shown to perform better than $\mathrm{BMI}$ and waist circumference in predicting all-cause mortality. ${ }^{9}$ Similar conclusions have been drawn from Tehran Lipid and Glucose Study except for WHpR in female population. ${ }^{10}$ 
A recent Brazilian epidemiological study suggested conicity index (C-Index) to equalise waist circumference and WHtR in identifying android pattern of fat excess and recommended its use. 11 Studies could not be found improved performance for $A B S I$ or $B R I$ in comparison to traditional measures like BMI, waist circumference and WHtR. 12 Contrasting findings are also related to gender in some studies as differing findings were observed by Wang et al., where the researchers observed ABSI performs better for male subjects and BRI and WHtR were demonstrated to be a better predictor heart disease among females. ${ }^{13}$

Based upon the promise that these new obesity measures bring in depicting underlying cardiovascular risk with varying literature on the subject and to improve clinical application in our set-up, a study was planned to evaluate the differences for these new vs old anthropometric measures among subjects with or without metabolic syndrome, diabetes, nephropathy, and dyslipidemia.

The second objective was to compare the diagnostic performance of these anthropometric measures by ROC curve analysis for risk prediction for aforementioned disease.

\section{METHODOLOGY}

This was a cross-sectional study which was carried out at the Departments of Pathology and Medicine at PNS Hafeez Hospital, Islamabad from January 2016 to December 2018. Formal Ethical Review Committee approval was sought for the study and Ethical Review Committee monitored the proceedings during the course of study. The target population were middle aged subjects of either gender, who presented at the Department of Medicine for annual medical evaluation without any prior history of known chronic disorder. Subjects with diabetes, ischemic heart disease, hypertension, age less than 18 or more than 70 years, having any other chronic or acute or chronic ailment, taking any medication at the time of study were excluded from the study. Subjects accepting the study participation proposal were requested to come to the Pathology Department in medical fasting status. Nonprobability convenience sampling was practised with up to 5 samples managed on 2-3 days of the week. At the time of presentation at Pathology Department, all subjects were explained regarding study requirements, kind of sampling requirements, and future use of data for research purpose with information about data confidentiality. All volunteering subjects were interviewed as per defined questionnaire followed by clinical and anthropometric examination. 14-19 Anthropometric measurements were taken as per standard formula.

Following the clinical examination, a total of $8-10 \mathrm{ml}$ blood was collected in EDTA, plain and Na-Fluoride bottles for lipids, fasting plasma glucose, ALT, uric acid,
$\mathrm{HbA1C}$ and insulin. Spot urine specimen for measuring urine albumin creatinine ratio (UACR). Measurements for total cholesterol, triglycerides and glucose by CHODPAP, GPO-PAP and GOD-PAP method. HbA1c was measured by "lon-exchange resin chromatography" method. Low density lipoprotein-cholesterol (LDLC) and high density lipoprotein cholesterol (HDLc) concentrations were measured by cholesterol esterase methodology using AVIDA 1800 clinical chemistry system. UACR was also measured by immunoturbidimetric method on ADVIA 1800 in 174 subjects. Serum insulin was measured by chemiluminescence method on Immulite ${ }^{\circledR}$ 1000. Insulin resistance was calculated using Mathew's et al. method i.e., homeostasis model assessment for Insulin resistance (HOMA-IR).

Subjects were classified as having metabolic syndrome or otherwise based upon IDF. Individuals having $\mathrm{HbA} 1 \mathrm{C}$ levels greater than $6.5 \%$ were diagnosed as having diabetes mellitus, while below that were considered non-diabetic. HOMAIR cut-off of 2.50 was utilised to categorise participants as having insulin resistance.

All data were added first into Excel and later shifted to IBM SPSS version-19. Descriptive statistics for differences between genders and anthropometric indices were calculated using independent sample t-test. Differences between anthropometric obesity indices between subjects with and without metabolic syndrome, diabetes and nephropathy were also evaluated using independent sample t-test. Pearson method was utilised to measure the correlation between lipid indices and obesity measures. ROC curve analysis was utilised to calculate the area under the curve (AUC) for various anthropometric indices keeping presence or absence of metabolic syndrome as state. A p-value of $<0.05$ was considered as significant.

\section{RESULTS}

The data included 110 males and 122 females with mean and 95\% confidence interval. Gender difference for age and various obesity anthropometric parameters are shown in Table I. All anthropometric obesity parameters showed significant differences between subjects with or without IDF defined metabolic syndrome $(p<0.001)$. Obesity indices including WHpR, BRI, C-Index and BAl did not show statistically significant results among subjects with or without diabetes as per $\mathrm{HbA} 1 \mathrm{C}$ cut-offs of $6.5 \%$, while BMI [ $(\mathrm{HbA} 1 \mathrm{c}<6.50(\mathrm{n}=181)=$ $26.68+5.4),(\mathrm{HbA} 1 \mathrm{c}>6.50 \quad(\mathrm{n}=47)=26.68+5.4)$, $\mathrm{p}=0.019]$, WHtR [(HbA1c $<6.50 \quad(\mathrm{n}=181=0.57+0.07)$, $(\mathrm{HbA1c}>6.50 \quad(n=47)=0.60+0.07), p=0.023]$, AVI $[(\mathrm{HbA} 1 \mathrm{c}<6.50(\mathrm{n}=181=17.56+3.86),(\mathrm{HbA} 1 \mathrm{c}>6.50$ $(n=47)=19.24+4.23), p=0.010], A B S I[(H b A 1 c<6.50$ $(n=181=1.82+0.58),(\mathrm{HbA} 1 \mathrm{c}>6.50 \quad(\mathrm{n}=47)=0.1 .58$ $+0.37), p=0.023$ ] showed significant differences. 
Nephropathy, as determined by UACR, was only found to be significant for BMI [(Group-1: Urine Albumin Creatinine Ratio $(U A C R)<2.5 \mathrm{mg} / \mathrm{g}(\mathrm{n}=135)=26.33+5.02$ and (Group-2: Urine Albumin Creatinine Ratio (UACR) $>2.4$ $\mathrm{mg} / \mathrm{g}(\mathrm{n}=39)=28.60+6.49)(\mathrm{p}=0.022)]$ and $A B S I[($ Group1: Urine Albumin Creatinine Ratio (UACR) $<2.5 \mathrm{mg} / \mathrm{g}$ $(n=135)=1.83+0.58$ and (Group-2: Urine Albumin Creatinine Ratio $(\mathrm{UACR})>2.4 \mathrm{mg} / \mathrm{g}(\mathrm{n}=39)=1.62+0.52)$ $(p=0.022)]$. No other anthropometric parameter was able to demonstrate significant difference to depict nephropathy.
Table II demonstrates Pearson's correlation between obesity measures and lipid indices. The pattern of correlation suggests highest correlation for $\mathrm{AVI}$ for most lipid indices followed by WHpR and WHtR, while the results for other obesity anthropometric parameters remain quite variable. Mean $A \cup C$ for obesity measures crossed 0.80 for WHtR and AVI, followed AUCs remain less than 0.800 for most other parameters. The least AUC i.e. 0.320, was observed for ABSI as depicted in Figure 1. The impact of obesity anthropometric indices

Table I: Gender differences in age and various traditional and new anthropometric indices.

\begin{tabular}{|c|c|c|c|c|c|c|}
\hline \multirow[t]{2}{*}{ Parameter } & \multirow[t]{2}{*}{ Gender } & \multirow[t]{2}{*}{$\mathrm{N}$} & \multirow[t]{2}{*}{ Mean } & \multicolumn{2}{|c|}{$95 \%$ Confidence Interval } & \multirow[t]{2}{*}{ Sig. (2-tailed) } \\
\hline & & & & Lower & Upper & \\
\hline \multirow[t]{2}{*}{ Age (years) } & Male & 110 & 47.98 & 45.83 & 50.20 & 0.068 \\
\hline & Female & 122 & 45.27 & 43.12 & 47.66 & \\
\hline \multirow[t]{2}{*}{ Body mass index (BMI) $\mathrm{Kg} / \mathrm{m}^{2}$} & Male & 110 & 25.98 & 25.14 & 26.96 & 0.002 \\
\hline & Female & 122 & 28.18 & 27.28 & 29.02 & \\
\hline \multirow[t]{2}{*}{ Waist to hip ratio (WHpR) } & Male & 110 & 0.93 & 0.90 & 0.94 & 0.171 \\
\hline & Female & 122 & 0.94 & 0.93 & 0.95 & \\
\hline \multirow[t]{2}{*}{ Waist to height ratio (WHtR) } & Male & 110 & 0.55 & 0.54 & 0.56 & 0.001 \\
\hline & Female & 122 & 0.60 & 0.59 & 0.61 & \\
\hline \multirow[t]{2}{*}{ Abdominal volume index (AVI) } & Male & 110 & 17.48 & 16.84 & 18.16 & 0.073 \\
\hline & Female & 122 & 18.39 & 17.58 & 19.12 & \\
\hline \multirow[t]{2}{*}{ Body roundness index (BRI) } & Male & 110 & 4.78 & 4.63 & 4.93 & 0.001 \\
\hline & Female & 122 & 5.80 & 5.60 & 5.98 & \\
\hline \multirow[t]{2}{*}{ Body adiposity index (BAI) } & Male & 110 & 26.93 & 25.94 & 27.92 & 0.001 \\
\hline & Female & 122 & 32.68 & 31.59 & 33.76 & \\
\hline \multirow[t]{2}{*}{ A body shape index (ABSI) } & Male & 110 & 1.92 & 1.81 & 2.02 & 0.001 \\
\hline & Female & 122 & 1.63 & 1.54 & 1.72 & \\
\hline \multirow[t]{2}{*}{ Conicity-index (C-Index) } & Male & 110 & 1.29 & 1.28 & 1.31 & 0.047 \\
\hline & Female & 122 & 1.31 & 1.30 & 1.33 & \\
\hline
\end{tabular}

Table II: Correlation between anthropometric indices and lipid indices.

\begin{tabular}{|c|c|c|c|c|c|c|}
\hline Anthropometric parameters & Total cholesterol & Fasting triglycerides & HDLc & LDLc & Non-HDLc & sdLDL^$^{\wedge}$ \\
\hline \multicolumn{7}{|l|}{ Body mass index (BMI) $\mathrm{Kg} / \mathrm{m}^{2}$} \\
\hline Pearson Correlation & $0.197^{\star *}$ & 0.115 & 0.126 & 0.032 & $0.139^{*}$ & 0.099 \\
\hline Sig. (2-tailed) & 0.003 & 0.081 & 0.056 & 0.626 & 0.035 & 0.132 \\
\hline \multicolumn{7}{|l|}{ Waist to hip ratio (WHpR) } \\
\hline Pearson Correlation & $0.205^{\star \star}$ & $0.173^{* *}$ & -0.004 & $0.169^{*}$ & $0.191^{* *}$ & $0.202^{* *}$ \\
\hline Sig. (2-tailed) & 0.002 & 0.008 & 0.957 & 0.010 & 0.004 & 0.002 \\
\hline \multicolumn{7}{|l|}{ Waist to height ratio (WHtR) } \\
\hline Pearson Correlation & $0.199^{* *}$ & $0.199^{\star *}$ & 0.083 & 0.070 & $0.157^{*}$ & $0.174^{\star *}$ \\
\hline Sig. (2-tailed) & 0.002 & 0.002 & 0.212 & 0.293 & 0.016 & 0.008 \\
\hline \multicolumn{7}{|l|}{ Abdominal Volume Index (AVI) } \\
\hline Pearson Correlation & $0.207^{* *}$ & $0.250^{* *}$ & -0.037 & 0.085 & $0.206^{* *}$ & $0.201^{* *}$ \\
\hline Sig. (2-tailed) & 0.002 & $<0.001$ & 0.580 & 0.201 & 0.002 & 0.002 \\
\hline \multicolumn{7}{|l|}{ Body roundness index (BRI) } \\
\hline Pearson Correlation & $0.167^{*}$ & 0.126 & $0.162^{*}$ & 0.047 & 0.099 & 0.123 \\
\hline Sig. (2-tailed) & 0.011 & 0.056 & 0.014 & 0.475 & 0.133 & 0.062 \\
\hline \multicolumn{7}{|l|}{ Body adiposity index (BAI) } \\
\hline Pearson Correlation & $0.140^{*}$ & 0.044 & $0.219^{* *}$ & 0.034 & 0.054 & 0.072 \\
\hline Sig. (2-tailed) & 0.032 & 0.505 & 0.001 & 0.604 & 0.415 & 0.274 \\
\hline \multicolumn{7}{|l|}{ A body shape index (ABSI) } \\
\hline Pearson Correlation & $-0.164^{*}$ & -0.100 & $-0.133^{*}$ & -0.016 & -0.105 & -0.095 \\
\hline Sig. (2-tailed) & 0.012 & 0.129 & 0.044 & 0.809 & 0.112 & 0.151 \\
\hline \multicolumn{7}{|l|}{ Conicity-index (C-Index) } \\
\hline Pearson Correlation & 0.085 & $0.235^{\star *}$ & -0.123 & 0.100 & b0.126 & $0.194^{* *}$ \\
\hline Sig. (2-tailed) & 0.200 & $<0.001$ & 0.062 & 0.131 & 0.055 & 0.003 \\
\hline
\end{tabular}

${ }^{\wedge}$ small dense $L D L$ cholesterol calculated as per Srisawasdi et al. for equation. 
on insulin resistance using general linear model (GLM) analysis, where the impact of various anthropometric indices was assessed as dependent variable keeping insulin resistance as fixed factor and gender as a covariate. The results showed that most indices including gender-adjusted AVI [Subjects with normal HOMAIR (17.04, 95\% Cl: 16.42-17.67) and high HOMAIR

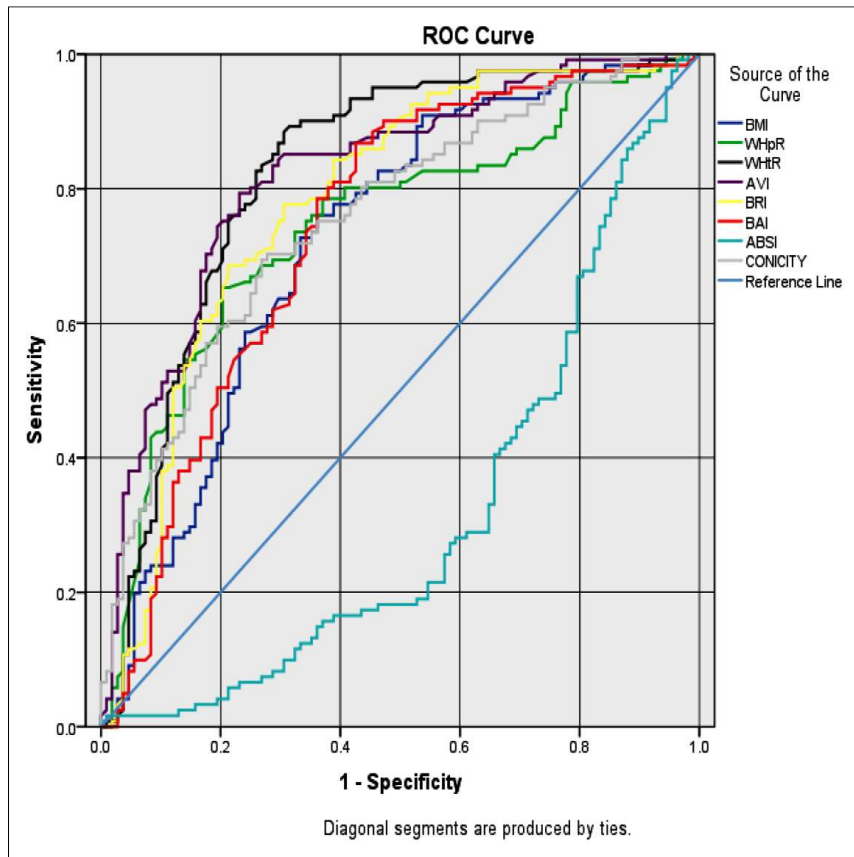

Figure 1: AUC for various anthropometric obesity measures diagnostic parameters for diagnosing gestational diabetes mellitus as: AUC WHtR= $0.823(95 \% \mathrm{Cl}: 0.766-0.880)$, AUC AVI=0.822(95\% Cl: $0.767-0.877), \mathrm{BRI}=$ 0.782 (95\% Cl: $0.720-0.844), A \cup C W H p R=0.744(95 \% \mathrm{Cl}: 0.679-0.809)$, AUC BAl $=0.740(95 \% \mathrm{Cl}: 0.674-0.806)$,AUC C-Index $=0.757(95 \% \mathrm{Cl}: 0.695$ $0.819)$, AUC BMI $=0.725(95 \% \mathrm{Cl}: 0.658-0.792)$ and $\mathrm{AUC} \mathrm{ABSI}=0.320(95 \%$ Cl: 0.249-0.391) $(p<0.001)$.

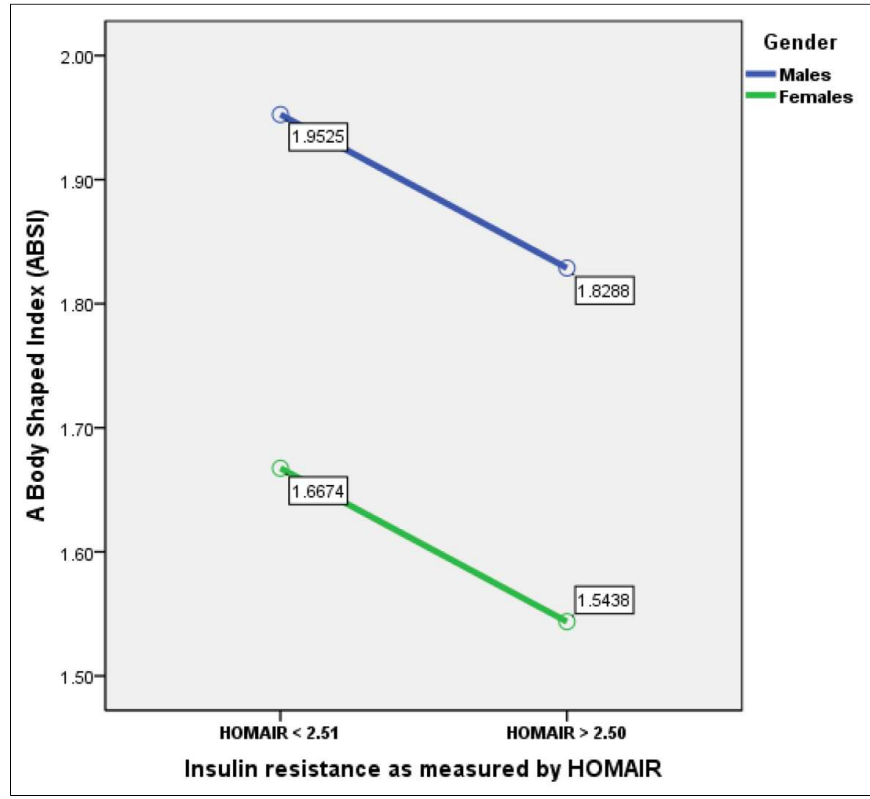

Figure 2: General Linear Model (GLM) showing the gender adjusted ABSI differences between subjects with normal HOMAIR $(1.89,95 \% \mathrm{Cl}$ : 1.79-2.00) and high HOMAIR $(1.61,95 \% \mathrm{Cl}: 1.51-1.70)$ for Body Mass Index (BMI) [Model significance $<0.001$ ]
(19.75, 95\% Cl: 18.87-20.60), model significance <0.001)], gender-adjusted BMI [Subjects with normal HOMAIR (26.37, 95\% Cl: 25.56-27.18) and high HOMAIR (28.61, $95 \% \mathrm{Cl}: 27.48-29.73)$, model significance $=0.002$ ], genderadjusted WHtR [subjects with normal HOMAIR (0.56, $95 \% \mathrm{Cl}: 0.55-0.57)$ and high HOMAIR $(0.60,95 \% \mathrm{Cl}$ : $0.59-0.62$ ), model significance $<0.001$ ], BRI [subjects with normal HOMAIR $(5.16,95 \% \mathrm{Cl}$ : 5.01-5.31) and high HOMAIR (5.58, 95\%Cl: 5.37-5.79), model significance= 0.002] and BAI [subjects with normal HOMAIR (29.28, $95 \% \mathrm{Cl}: 28.36-30.31)$ and high HOMAIR $(31.06,95 \% \mathrm{Cl}$ : 29.75-30.31), model significance $=0.031$ ] showed a significant rise in insulin resistance with increase in obesity measures. However, C-Index did not show the results to be significant as [Subjects with normal HOMAIR (1.29, 95\%Cl: 1.28-1.30) and high HOMAIR (1.33, 95\% Cl: $1.31-1.34)$, model significance $=0.086]$. Alongside, we also observed that ABSI levels dropped in both genders with rise in insulin resistance. (Figure 2).

\section{DISCUSSION}

The study evaluated three conventional obesity anthropometric indices including $\mathrm{BMI}, \mathrm{WHpR}$ and $\mathrm{WHtR}$; and 5 new obesity measures which included AVI, BRI, BAI, $A B S I$ and $C$-Index. These indices can be divided mainly into weight included measures (BMI, ABSI and C-Index) and weight excluded measures $(\mathrm{WHpR}, \mathrm{WHtR}, \mathrm{AVI}$, $\mathrm{BRI}$, and $\mathrm{BAI}$ ). This study has shown that most of the evaluated parameters namely BMI, WHtR, WHpR, AVI, $\mathrm{BRI}, \mathrm{BAI}, \mathrm{ABSI}$ and C-Index are associated variably with various in-vogue biochemically defined risk factors including dyslipidemia, insulin resistance and nephropathy.

Prime example for negative associations between invogue anthropometric obesity measures included WHpR, which was not related with diabetes, nephropathy or gender status. The probable explanation for this relates to the denominator effect which changes with body frame. This aspect has been highlighted in other studies and also literature review has suggested $\mathrm{WHpR}$ to be less useful than other anthropometric obesity markers. ${ }^{4,9,3}$ Most studies have highlighted less utility of WHpR in relation to other studies, though authorities still recommend its use in various clinical set-ups.9,7 Similarly, conicity index inclusive of parameters, like height, weight and waist, have only been found useful to predict metabolic syndrome. Results for differences in diabetes, nephropathy, and insulin resistance and poor correlation with most lipid indices along with the extensive mathematical calculation makes it less feasible and clinically predictive obesity anthropometric marker. Though some studies support its use in predicting risk in population, 10,11 still contrasting literature in line with study observations seem to make this anthropometric index less useful than traditional measures as depicted in the Chinese study. 20 Zhang et al. found conicity index 
to be only useful once adjusted with total body fat imply indirectly the lesser utility of $\mathrm{C}$-index in predicting underlying metabolic disease risk. ${ }^{21}$

All anthropometrics, whether conventional or the emerging ones, rely upon specific patient characteristics like fat mass, visceral fat mass or muscle mass. Strulov et al. discussed the high race, and gender and age variability between individuals resulting in differences in body composition. ${ }^{22}$ Moreover, the other variable is the mathematical model dealing with how multiple patient anthropometric data is fitted into equation along with certain constants. O'Neill et al. attributed various obesity measures to suffer due to non-availability of gold standard to define obesity; leading almost $45 \%$ white females are misclassified as non-obese resulting in various false negative and false positive. ${ }^{23}$

In the opinion of the authors, the useful marker for clinical use will depend upon what element of metabolic disease we need to predict. It seems that WHtR and AVI better predicts the visceral fat accumulation. Our observations suggest conicity index and $\mathrm{WHpR}$ provide less useful information than other obesity measures. Similarly, ABSI has shown the lowest AUC highlighting this to be least useful for our population. In this regard, the study results of Biolo et al. have also indicated an inverse relationship between ABSI and fat-free mass; and can act as surrogate marker for lean mass rather fat-mass. ${ }^{24}$ The same author highlighted its use for predicting sarcopenia. Finally, the study by Caitano Fontela et al. has concluded that the use of anthropometric parameters cannot be independent predictor of underlying coronary artery disease. 25 Therefore, the anthropometry-based obesity measures must be correlated with biochemical and clinical findings to actually understand underlying risk for any metabolic disease including atherosclerotic cardiovascular diseases (ASCVD).

Certain limitations to this study must be acknowledged. It was a hospital-based cross-sectional study and the findings must be replicated at a wider scale through a prospective epidemiological study to establish the link between candidate obesity markers and metabolic diseases. Furthermore, caution must be exercised while evaluating subjects for cardio fitness or deciding on matters of metabolic disease predisposition based upon only one parameter; as these factors are just dependent ones and needs to be related with biochemical or clinical findings to attribute risk for a particular metabolic disease.

This study is an important clinical addition in terms of providing regional data on various emerging anthropometry-based obesity indices in the presence of conventional parameters like BMI, WHpR and WHtR and, therefore, provides real insight about their use in terms of predicting a disease. The variable information upon these anthropometry-based parameters is quite significant as one obesity marker may just not imply an underlying disease or presence of excessive visceral fat; and thus overall clinical picture needs to be understood rather than decision being based upon these parameters. Moreover, obesity patterns in our society may be quite subtle to allow excessive fat accumulation in a very variable way to result CVD morbidity and mortality thus warranting further research on this aspect of Asian obesity paradox.

\section{CONCLUSION}

Different anthropometry obesity indices aim to detect different aspect of metabolic diseases. WHpR and conicity index may not be potent to provide enough information to predict underlying metabolic disease. WHtR and AVI have the highest AUC to diagnose metabolic syndrome and were better associated with multiple metabolic diseases. These anthropometric obesity markers are not independent factors and thus accurate risk prediction should be based upon the combined use of suggested anthropometric markers, biochemical data, and clinical information.

\section{ETHICAL APPROVAL:}

The study had prior formal approval of hospital's Ethical Review Committee.

\section{PATIENTS' CONSENT:}

The participants of the study signed written informed consents before inclusion into study.

\section{CONFLICT OF INTEREST:}

Authors declared no conflict of interest.

\section{AUTHORS' CONTRIBUTION:}

SHK: Conceptualisation of study, laboratory testing, SPSS data analysis, final manuscript preparation.

RS: Patient's anthropometric and clinical evaluation, contribution towards results preparation and writing.

NF: Patient clinical evaluation, data analysis and contribution to result analysis and discussion.

Al: Overall guidance, data analysis and manuscript writing. All authors finally approved the study.

\section{REFERENCES}

1. Wright SM, Aronne LJ. Causes of obesity. Abdom Imaging 2012; 37:730-2.

2. Yu JY, Choi WJ, Lee HS, Lee JW. Relationship between inflammatory markers and visceral obesity in obese and overweight Korean adults: An observational study. Medicine (Baltimore) 2019; 98:e14740.

3. Müller MJ, Braun W, Enderle J, Bosy-Westphal A. Beyond BMI: Conceptual issues related to overweight and obese patients. Obes Facts 2016; 9:193-205.

4. Bell RA, Chen H, Saldana S, Bertoni AG, Effoe VS, Hairston KG, 
et al. Comparison of measures of adiposity and cardiovascular disease risk factors among african american adults: The jackson heart study. J Racial Ethn Health Disparities 2018; 5:1230-7.

5. Gruson E, Montaye M, Kee F, Wagner A, Bingham A, Ruidavets $\mathrm{JB}$, et al. Anthropometric assessment of abdominal obesity and coronary heart disease risk in men: The PRIME study. Heart 2010; 96:136-40.

6. Veghari G, Salehi A, Vaghari M. The comparison of waist circumference, waist-to-hip ratio, and waist-to-height ratio among rural women adults in the north of Iran, between the years 2004 and 2013. ARYA Atheroscler 2018; 14:169-76.

7. Perona JS, Schmidt Rio-Valle J, Ramírez-Vélez R, CorreaRodríguez M, Fernández-Aparicio Á, González-Jiménez E. Waist circumference and abdominal volume index are the strongest anthropometric discriminators of metabolic syndrome in Spanish adolescents. Eur J Clin Invest 2019; 49:e13060.

8. Tian S, Zhang X, Xu Y, Dong H. Feasibility of body-roundness index for identifying a clustering of cardiometabolic abnormalities compared to BMI, waist circumference and other anthropometric indices: The China Health and Nutrition Survey, 2008 to 2009. Medicine (Baltimore) 2016; 95:e4642.

9. Ji M, Zhang S, An R. Effectiveness of a body shape index (ABSI) in predicting chronic diseases and mortality: A systematic review and meta-analysis. Obes Rev 2018; 19:737-59.

10. Sardarinia M, Ansari R, Azizi F, Hadaegh F, Bozorgmanesh M. Mortality prediction of a body shape index versus traditional anthropometric measures in an Iranian population: Tehran lipid and glucose study. Nutrition 2017; 33:105-112.

11. Filgueiras MS, Vieira SA, Fonseca PCA, Pereira PF, Ribeiro AQ, Priore SE, et al. Waist circumference, waist-to-height ratio and conicity index to evaluate android fat excess in Brazilian children. Public Health Nutr 2019; 22:140-6.

12. Chang Y, Guo X, Chen Y, Guo L, Li Z, Yu S, et al. A body shape index and body roundness index: Two new body indices to identify diabetes mellitus among rural populations in northeast China. BMC Public Health 2015; 15:794.

13. Wang F, Chen Y, Chang Y, Sun G, Sun Y. New anthropometric indices or old ones: Which perform better in estimating cardiovascular risks in Chinese adults. BMC Cardiovasc Disord 2018; 18:14.
14. National Health and Nutrition Examination Survey (NHANES). Anthropometry Procedures Manual. Available at:- https://www. cdc.gov/nchs/data/nhanes/nhanes_07_08/manual_an.pdf. Retrieved on: 19-March-2019.

15. Guerrero-Romero F, Rodríguez-Morán M. Abdominal volume index. An anthropometry-based index for estimation of obesity is strongly related to impaired glucose tolerance and type 2 diabetes mellitus. Arch Med Res 2003; 34:428-32.

16. Thomas DM, Bredlau C, Bosy-Westphal A, Mueller M, Shen W, Gallagher $\mathrm{D}$, et al. Relationships between body roundness with body fat and visceral adipose tissue emerging from a new geometrical model. Obesity (Silver Spring) 2013; 21:2264-71.

17. Bergman RN, Stefanovski D, Buchanan TA, Sumner AE, Reynolds JC, Sebring NG, et al. A better index of bodyadiposity. Obesity (Silver Spring). 2011; 19:1083-9.

18. Krakauer NY, Krakauer JC. A new body shape index predicts mortality hazard independently of body mass index. PLoS One 2012; 7:e39504.

19. Valdez R. A simple model-based index of abdominal adiposity. $J$ Clin Epidemiol 1991; 44:955-6.

20. Chen X, Liu Y, Sun X, Yin Z, Li H, Deng K, et al. Comparison of body mass index, waist circumference, conicity index, and waist-to-height ratio for predicting incidence of hypertension: The rural Chinese cohort study. J Hum Hypertens 2018; 32:228-35.

21. Zhang Y, Zeng Q, Li X, Zhu P, Huang F. Application of conicity index adjusted total body fat in young adults - a novel method to assess metabolic diseases risk. Sci Rep 2018; 8: 10093.

22. Strulov Shachar S, Williams GR. The obesity paradox in cancermoving beyond BMI. Cancer Epidemiol Biomarkers Prev 2017; 26:13-6.

23. O'Neill D. Measuring obesity in the absence of a gold standard. Econ Hum Biol 2015; 17:116-28.

24. Biolo G, Di Girolamo FG, Breglia A, Chiuc M, Baglio V, Vinci P. et al. Inverse relationship between "a body shape index" (ABSI) and fat-free mass in women and men: Insights into mechanisms of sarcopenic obesity. Clin Nutr 2015; 34:323-7.

25. Caitano Fontela P, Winkelmann ER, Nazario Viecili PR. Study of conicity index, body mass index and waist circumference as predictors of coronary artery disease. Rev Port Cardiol 2017; 36:357-64. 